\title{
LETTER \\ Impact of Viewing Distance on Task Performance and Its Properties
}

\author{
Makio ISHIHARA $^{\dagger \mathrm{a})}$, Member and Yukio ISHIHARA ${ }^{\dagger \dagger \mathrm{b})}$, Nonmember
}

SUMMARY This paper discusses VDT syndrome from the point of view of the viewing distance between a computer screen and user's eyes. This paper conducts a series of experiments to show an impact of the viewing distance on task performance. In the experiments, two different viewing distances of $50 \mathrm{~cm}$ and $350 \mathrm{~cm}$ with the same viewing angle of 30 degrees are taken into consideration. The results show that the long viewing distance enables people to manipulate the mouse more slowly, more correctly and more precisely than the short.

key words: viewing distance, physical fatigue, physiological stress, task performance, VDT syndrome

\section{Introduction}

VDT syndrome is a symptom that is caused by extensive use of eyes for long hours and pains in the neck or shoulders due to sitting at desks for long periods of time, and physiological stress that stems from monotonous and continuous work requiring no errors etc. In 2008, the Japanese Ministry of Health, Labor and Welfare conducted an investigation into VDT syndrome and the report said that the percentage of people in Japan who use computers at work is $86.2 \%$. Among them, the ratio of people who use computers for more than two hours is $62.6 \%$, and the ratio of people who claim to have dry eyes, stiff neck, lumbago, headache, and dizziness is $78.0 \%$. Thus VDT syndrome should be addressed as carefully and quickly as possible.

To deal with VDT syndrome, A. Uetake et al. [1] discussed an evaluation method of eye fatigue during VDT tasks. They found that eye fatigue is expressed as a function of pupil diameter and focusing strength. T. Katayama et al. [2] investigated the impact of color patterns displayed on a screen on work performance and fatigue. They found that an appropriate contrast between characters and background tends to alleviate much more fatigue. Y. Kato et al. [3] built a posture feedback neck band to alleviate pain in the neck and shoulders. Users wear the band on their neck and the band rings an alarm when their posture becomes misaligned during VDT tasks.

In regard to human ergonomics, international standards of ISO9241-303 specify requirements for output devices, es-

Manuscript received June 4, 2018.

Manuscript publicized July 2, 2108.

${ }^{\dagger}$ The author is with Fukuoka Institute of Technology, Fukuokashi, 811-0295 Japan.

${ }^{\dagger}$ The author is with Shimane University, Matsue-shi, 690-8504 Japan.

a) E-mail: m-ishihara@ fit.ac.jp

b)E-mail: iyukio@ipc.shimane-u.ac.jp

DOI: 10.1587/transinf.2018EDL8117 pecially for electronic visual displays. It says for example that the viewing distance, which refers to the physical distance between the surface screen and the user's eyes, should be 30 to $40 \mathrm{~cm}$ for children and young people, and more than $40 \mathrm{~cm}$ for adults and older people. Though a lot of approaches and efforts from various points of view have been made to address VDT syndrome over the years, it still remains a problem for many office workers.

As mentioned, optimal viewing distances have been discussed often from the side of human ergonomics but not task performance. Previously, the authors [4] conducted a pilot experiment on the relation between viewing distances and task performance. The result implies that the task performance improves when the viewing distance is longer. The aim of this manuscript is to confirm an impact of viewing distances on task performance by experiment and discuss its properties.

Section 2 defines three hypotheses to confirm the impact of viewing distances on task performance. Section 3 conducts a series of experiments along with the hypotheses. Section 4 shows the result and Sect. 5 confirms the impact of viewing distances on task performance by evaluation of the hypotheses. Finally, Sect. 6 gives the concluding remarks.

\section{Research Hypotheses}

Based on the result of our pilot experiment [4], this manuscript confirms the impact of viewing distances on task performance through a series of experiments with a mouse manipulation task and a calculation task, taking their speed and accuracy into consideration. This manuscript focuses on three hypotheses for confirmation of the impact. The hypotheses $\mathbf{H 1}$ and $\mathbf{H 2}$ are the primary claims of this manuscript and $\mathbf{H 3}$ is a supporting claim for $\mathbf{H 1}, \mathbf{H 2}$.

H1 The longer viewing distance enables people to perform mouse manipulation more slowly and more correctly.

H2 The longer viewing distance enables people to perform calculation more slowly and more correctly.

H3 The longer viewing distance enables people to experience less physiological stress.

\section{Experiment}

Figure 1 shows our experimental settings. In the experiment, two viewing distances are taken into consideration: $50 \mathrm{~cm}$ and $350 \mathrm{~cm}$. The horizontal viewing angle for both 


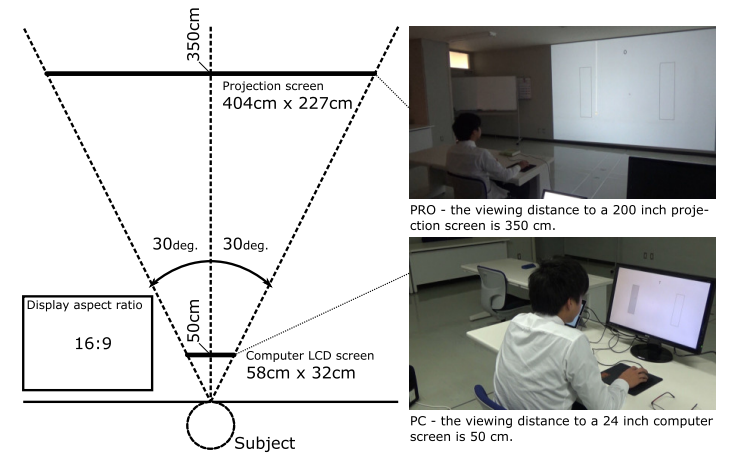

Fig. 1 Experimental settings.

the viewing distances is set to 30 degrees, which is defined in ergonomics of human system interaction in ISO9241-303. Thus a 24 inch computer LCD screen $(58 \times 32 \mathrm{~cm}, 59 \mathrm{~Hz})$ is used for the viewing distance of $50 \mathrm{~cm}$ and a 200 inch projection screen $(404 \times 227 \mathrm{~cm}, 48 \mathrm{~Hz})$ for $350 \mathrm{~cm}$. The vertical viewing angle is 17.8 degrees for the LCD screen and 18.0 degrees for the projection screen. The former condition is referred to by PC and the later by PRO. Note that contents on both the screens are the same but they are enlarged on the projection screen so they appear the same in size in the subject's eyes. It should also be mentioned that there is a difference between the lighting systems of the LCD/projection screens: the emitted light and the reflected light. As it could affect the result, this paper uses questionnaires specified in ISO9241-411 to assess physical fatigue of the subject's neck, eyes etc.

The subjects' heart rate (RRI) is measured to assess their physiological stress level by Union Tool WHS-1. By carrying out a spectral analysis of the given RRI sequence, the ratio of LF (the spectral integration at 0.05 to $0.15 \mathrm{~Hz}$ ) to $\mathrm{HF}$ (the spectral integration at 0.15 to $0.40 \mathrm{~Hz}$ ) is used to express an index of physiological stress: relax $(0.0 \sim 0.8)$ and high physiological stress (2.0 5.0) according to Fatigue Science Laboratory Inc. A moving window of 60 seconds is employed to carry out the analysis and observe changes of the index of $\mathrm{LF} / \mathrm{HF}$ per second through the experiment.

The heart rate is measured through the experiment but only the part for Kraepelin test is used for evaluation because the change of physiological stress appears on the spectrum with a temporal delay of 2.5 to 20 seconds at minimum, depending on the target frequency of 0.05 to $0.40 \mathrm{~Hz}$. So, a certain period of time for subjects to perform the test is required to obtain stable data. The expected elapsed time for Kraepelin test is 15 minutes and it is long enough to obtain data but the elapsed time for Tapping test is not long enough.

\subsection{Tasks and Subjects}

Each subject performs two tasks, Tapping test and Kraepelin test at each condition: PC and PRO. Tapping test measures accuracy and speed of mouse manipulation. Figure 2 (left) shows a look of Tapping test. Two rectangles are placed on the left and right of the screen separately and the sub-

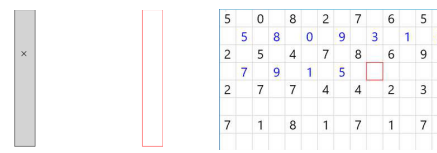

Fig. 2 Tapping test (left) and Kraepelin test (right).

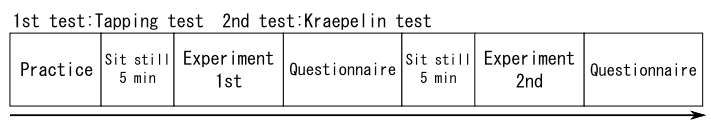

Fig. 3 Procedure of the experiment.

jects are asked to move the mouse cursor to either rectangle and click on it. Next the subject moves the cursor to the other rectangle and clicks on it alternating 50 times in total as quickly and correctly as possible. During the experiment, the elapsed time to complete the test and the number of misclicks in total are recorded.

Kraepelin test measures accuracy and speed of addition of a 1-digit number to a 1-digit number. Figure 2 (right) shows a look of Kraepelin test. An array of 1-digit numbers is displayed and the subjects are asked to add a number to the adjacent number from left to right and answer the first digit of the obtained value. The subjects keep doing calculations for 15 minutes as many and correctly as possible. During the experiment, the elapsed time to complete the test and the number of wrong answers are recorded.

There were 22 subjects aged from 21 to 23 . All were right-handed and had experience with manipulating a computer mouse. They also had good eyesight.

\subsection{Experiment Procedure}

Figure 3 shows a procedure of our experiment. Each subject performs Tapping test first, which is followed by Kraepelin test. The subjects have a practice time to become familiar with each test before it begins. After that, they sit still for 5 minutes then start the test at either condition PC or PRO and fill out a questionnaire about their physical fatigue. They sit still for another 5 minutes then start the test again at the other condition and fill out the questionnaire. The order of conditions between PC and PRO is balanced among subjects to remove order effects. Each subject performs one trial at each condition for each test, resulting in

$$
\begin{aligned}
& 22 \text { subjects } \times 2 \text { tests } \times 2 \text { conditions } \times 1 \text { trial } \\
& =88 \text { trials in total. }
\end{aligned}
$$

\section{Results}

\subsection{Tapping Test}

Figure 4 (left) shows accuracy of the mouse manipulation performed in Tapping test. The horizontal axis shows PC and PRO conditions, and the vertical one shows a percentage of the misclicks recorded. Each line represents a different subject and the thick dashed line represents the average 

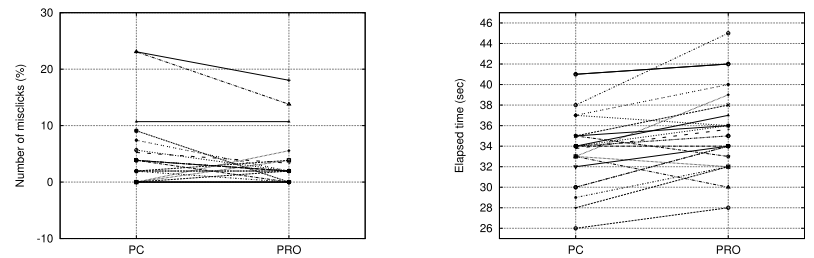

Fig. 4 The percentage of misclicks during Tapping test (left) and the elapsed time to complete the test (right).
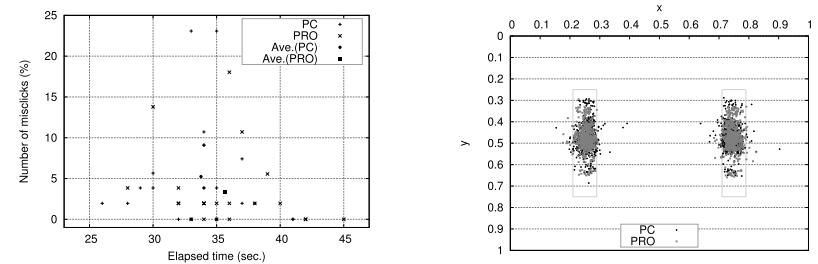

Fig.5 The scattered diagram between elapsed time and misclicks (left) and all clicks including misclicks during Tapping test (right).

across all the subjects. The average of the misclicks at PC is $5.24 \%$ and $3.34 \%$ at PRO, and the standard deviation is $6.61 \%$ and $4.78 \%$, respectively. The paired t test confirmed that there is a significant impact of the viewing distances on accuracy of the mouse manipulation $[\mathrm{t}(21)=2.26$ at $\mathrm{p}=.05]$.

Figure 4 (right) shows speed of the mouse manipulation. The vertical axis shows the elapsed time to complete the test in second. The average of the elapsed time is 33.77 seconds for PC and 35.64 seconds for PRO, and the standard deviation is 3.77 and 4.11 , respectively. The paired $t$ test confirmed that there is a significant impact of the viewing distances on speed of the mouse manipulation $[\mathrm{t}(21)=-3.59$ at $\mathrm{p}=.05]$.

Figure 5 (left) shows the scattered diagram for relationships between the elapsed time and the number of the misclicks. In the diagram, dots for PC tend to be on the upper left relatively to those for PRO and it shows that people performs the test slowly and correctly for PRO comparatively with PC.

Figure 5 (right) shows all the clicks including the misclicks across all the subjects. Black points represent those for PC and gray for PRO. The scale of horizontal axis or $x$ and vertical one or $y$ is normalized into 0 to 1 . The origin $(0,0)$ is the top left corner. For the left box, the average of the clicks is 0.259 in PC and 0.254 in PRO, and the variance is 0.00043 and 0.00033 , respectively. For the right box, the average is 0.742 in $\mathrm{PC}$ and 0.745 in PRO, and the variance is 0.00046 and 0.00035 , respectively. The $f$ test confirmed that there is a significant impact of the viewing distances on horizontal variance of the clicks for the left box $[\mathrm{f}(582,566)=1.313$ at $\mathrm{p}=.01]$ and the right $[\mathrm{f}(578,573)=1.312$ at $\mathrm{p}=.01]$.

\subsection{Kraepelin Test}

Figure 6 (left) shows accuracy of the calculation performed in Kraepelin test. The vertical axis shows a percentage of
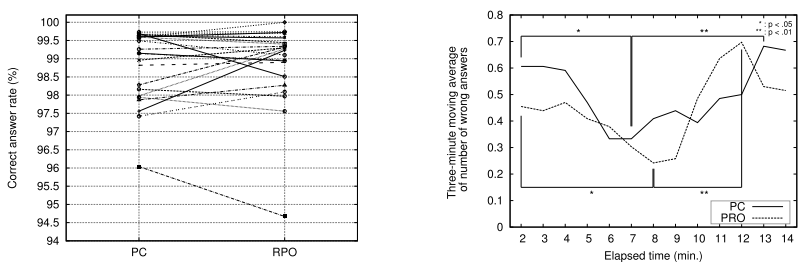

Fig. 6 The percentage of correct answers during Kraepelin test (left) and the 3-minute moving average of number of wrong answers (right).
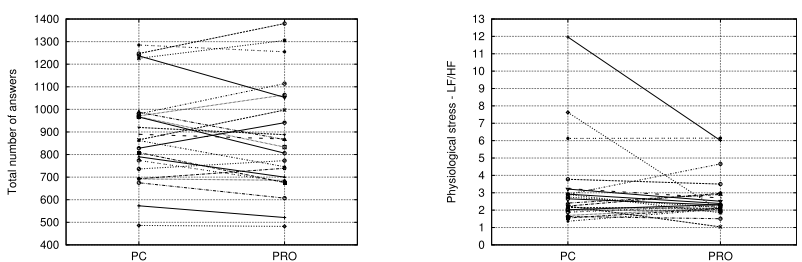

Fig. 7 The total number of answers (left) and the change of physiological stress levels (right) during Kraepelin test.

the correct answers. The average of the correct answers is $98.82 \%$ for PC and $98.90 \%$ for PRO, and the standard deviation is $1.01 \%$ and $1.14 \%$, respectively. Statistically, an impact of the viewing distances on accuracy of the calculation is not confirmed.

Figure 6 (right) shows change in accuracy of calculation during the test. The horizontal axis shows the elapsed time and the vertical shows the three-minute moving average of the number of wrong answers across all the subjects. The solid line represents PC and the dashed one does PRO. As for PRO, the paired t test shows that there is a significant difference in accuracy of the calculation between 2minute and 8-minute elapsed time [ $\mathrm{t}(65)=2.026$ at $\mathrm{p}=.05]$ and there is also a significant difference between 8 -minute and 12-minute elapsed time [ $\mathrm{t}(65)=-3.098$ at $\mathrm{p}=.01]$. Similarly the $\mathrm{t}$ test for PC shows that there is a significant difference in accuracy of the calculation between 2-minute and 7-minute elapsed time $[\mathrm{t}(65)=2.181$ at $\mathrm{p}=.05]$, and 7 -minute and 13-minute elapsed time $[\mathrm{t}(65)=-3.197$ at $\mathrm{p}=.01]$.

Figure 7 (left) shows speed of the calculation. The vertical axis shows the total answers during the calculation test. The average of the total answers is 889.5 for PC and 868.7 for PRO, and the standard deviation is 218.3 and 246.5, respectively. Statistically, an impact of the viewing distances on speed of the calculation is not confirmed.

\subsection{Physiological Stress}

Figure 7 (right) shows the result of an influence on physiological stress obtained from Kraepelin test. The vertical axis shows the average of the obtained indexes of LF/HF from each subject over the test at each condition. Each line represents a different subject and the thick line represents the average across all the subjects. Note that data from one subject was corrupted, so that it was removed from evaluation. The average at PC condition is 3.24 and it is 2.70 at PRO condition, and the standard deviation is 6.25 and 1.80 , re- 


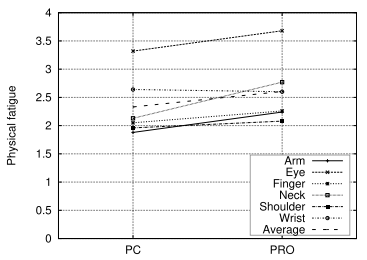

Fig. 8 The change of physical fatigue levels.

spectively. Statistically, an impact of the viewing distances on physiological stress is not confirmed.

\subsection{Physical Fatigue}

Figure 8 shows the result of a questionnaire about the subjects' physical fatigue on fingers, wrists, arms etc. from Tapping and Kraepelin tests. The vertical axis shows the average of the obtained physical fatigue levels from 0 to 10 across all the subjects and the two tests. The average of the physical fatigue levels is 2.47 for PC and 2.71 for $\mathrm{PRO}$, and the standard deviation is 2.26 and 2.33 , respectively. The paired $t$ test confirmed that there is a significant impact of the viewing distances on the physical fatigue $[\mathrm{t}(263)=-2.87$ at $\mathrm{p}=.05]$.

Looking at the physical fatigue on the neck, it has a comparatively large increase in PRO by comparison with PC. The paired $t$ test confirmed that there is a significant impact of the viewing distances on the physical fatigue on the neck $[\mathrm{t}(43)=-2.57$ at $\mathrm{p}=.05]$. The impact on the eyes seems to be large but it was not confirmed.

\section{Evaluation}

This section evaluates three hypotheses defined in Sect. 2 by overviewing the results obtained from the experiments.

For the first hypothesis H1, the results from Tapping test show statistically that the long viewing distance enables people to manipulate the mouse cursor more slowly (the elapsed time was extended by 1.87 seconds) and more correctly (the number of the misclicks was decreased by $1.9 \%$ ), and more precisely (the horizontal variance of the clicks on targets was decreased by 0.00010 , that is 0.19 pixel for a Full HD resolution $1920 \times 1080$ for the left target and 0.00011 , that is 0.21 pixel for the right) than the short viewing distance. These results support $\mathbf{H 1}$.

For the second hypothesis $\mathbf{H 2}$, the results from Kraepelin test do not show any strong evidence for the hypothesis but to some extent there seems to be a tendency towards the hypothesis. As regards change of accuracy of calculation during the test, the results show statistically that the accuracy drops at the beginning and rises almost double in the middle for both the viewing distances while the accuracy for the long viewing distance seems to be mostly better than the short. A further experiment is required to reinforce the hypothesis. The results are not enough to support $\mathbf{H 2}$.

For the third hypothesis $\mathbf{H 3}$ for physiological stress, the results from the spectral analysis of RRIs do not show any strong evidence to support the hypothesis while they show a certain tendency towards the hypothesis. A further experiment is required to reinforce it and clarify the underlying factors in H1, H2. The results are not enough to support H3.

For an influence of physical fatigue over the experiments, the questionnaires' result shows statically that the long viewing distance makes people suffer from physical fatigue more severely (the stress level was increased by 0.24 points) than the short. Particularly, the fatigue on the neck is severely increased for the long viewing distance. It could be because the subjects' face angle was slightly downward for the short viewing distance while it was upward for the long. As regards lighting systems, the fatigue on the eyes is comparatively large for both the viewing distances but the impact of the viewing distances on the eyes is not confirmed.

Above all, the fatigue on the neck might affect the results. To ease the fatigue on the neck, the face angle of the subject should be carefully designed by lowering the projection screen, leading to the use of head-mounted displays. In that case the fatigue on the eyes is likely to be severer and the fatigue on the neck could be considerable due to the weight of the device. A further experiment is required after the experiment settings are carefully designed based on the obtained findings mentioned above.

Finally the impact of viewing distances on task performance is confirmed partly by $\mathbf{H 1}$.

\section{Conclusion}

This paper discussed the impact of viewing distances on task performance and conducted a series of experiments with the two tasks. The results showed that longer viewing distances enable people to manipulate a mouse more slowly, correctly and precisely. The impact was partly confirmed.

\section{Acknowledgments}

This work was supported by Computer Science Laboratory of Fukuoka Institute of Technology.

\section{References}

[1] A. Uetake, M. Otsuka, Y. Takasawa, and A. Murata, "On evaluation index for visual fatigue induced during a VDT task," IEICE Trans. Fundamentals (Japanese Edition), vol.J83-A, no.12, pp.1521-1529, Dec. 2000.

[2] T. Katayama, S. Shoyama, and Y. Tochihara, "Effects of blue background in the negative display mode on VDT work efficiency and fatigue," Journal of Human and Living Environment, vol.22, no.1, pp.29-38, May 2015 (in Japanese).

[3] Y. Kato, S. Fukuda, Y. Suzuki, and S. Ota, "Effect of posture feedback band on posture alignment and pain during a visual display terminal task," Journal of the Japanese Society for Experimental Mechanics, vol.16, no.4, pp.315-319, 2017 (in Japanese).

[4] M. Ishihara and Y. Ishihara, "A pilot study on impact of viewing distance to task performance," Proc. 2017 International Conference on Biometrics and Kansei Engineering, pp.139-143, IEEE, 2017. 